\title{
Water-side turbulence enhancement of ozone deposition to the ocean
}

\author{
C. W. Fairall ${ }^{1}$, D. Helmig ${ }^{2}$, L. Ganzeveld ${ }^{3}$, and J. Hare , $^{4}$ \\ ${ }^{1}$ NOAA Earth Science Research Laboratory, Boulder, CO, USA \\ ${ }^{2}$ INSTAAR, University of Colorado, Boulder, CO, USA \\ ${ }^{3}$ Max-Planck Institute for Chemistry, Mainz, Germany \\ ${ }^{4}$ CIRES, University of Colorado, Boulder, CO, USA \\ *now at: SOLAS International Project Office, University of East Anglia, Norwich, UK
}

Received: 3 March 2006 - Published in Atmos. Chem. Phys. Discuss.: 26 June 2006

Revised: 4 December 2006 - Accepted: 15 January 2007 - Published: 25 January 2007

\begin{abstract}
A parameterization for the deposition velocity of an ocean-reactive atmospheric gas (such as ozone) is developed. The parameterization is based on integration of the turbulent-molecular transport equation (with a chemical source term) in the ocean. It extends previous work that only considered reactions within the oceanic molecular sublayer. The sensitivity of the ocean-side transport to reaction rate and wind forcing is examined. A more complicated case with a much more reactive thin surfactant layer is also considered. The full atmosphere-ocean deposition velocity is obtained by matching boundary conditions at the interface. For an assumed ocean reaction rate of $10^{3} \mathrm{~s}^{-1}$, the enhancement for ozone deposition by oceanic turbulence is found to be up to a factor of three for meteorological data obtained in a recent cruise off the East Coast of the U.S.
\end{abstract}

\section{Introduction}

The transport, formation and depletion of ozone have received significant research attention because of the recognized importance of ozone for the chemical and radiative properties of the atmosphere. Ozone is the most important precursor of the $\mathrm{OH}$ radical in the troposphere. Both ozone and $\mathrm{OH}$ are fundamental for the oxidizing capacity of the atmosphere and their concentrations determine the removal rates of many atmospheric contaminants. Increased anthropogenic emissions of nitrogen oxides and hydrocarbons, both being precursors of photochemical ozone production in the atmosphere, have led to significant increases in global, surface-level ozone concentrations. It has been estimated that tropospheric ozone has at least doubled since preindustrial times (Lamarque et al., 2005). Observations from background monitoring sites indicate that ozone continues

Correspondence to: C. W. Fairall

(chris.fairall@noaa.gov) to rise (Oltmans et al., 1998; Vingarzan, 2004; Helmig et al., 2007). Previous and anticipated future increases in background tropospheric ozone are a concern for several reasons. Ozone is a toxin to humans and animal life on Earth. Furthermore, tropospheric ozone has a significant $(\sim 13 \%)$ contribution to anthropogenic greenhouse gas forcing (IPCC, 2001), which possibly might further increase in the future due to continued increases in ozone and concomitant reductions in the growth rates of other important greenhouse gas emissions. These unique roles of ozone in atmospheric chemistry have motivated a plethora of research on improving our understanding of formation, transport and loss processes of atmospheric ozone.

Ozone deposition to the oceans represents a significant loss from the atmosphere; however, atmospheric and oceanic processes that determine the uptake of ozone to surface water are poorly understood. An accepted model for the description of dry deposition relies on the resistance approach (Wesely and Hicks, 2000). Deposition is expressed quantitatively by the deposition velocity $\left(V_{d}\right)$, which depends on different resistance terms, with $V_{d}=\left(R_{a}+R_{b}+R_{c}\right)^{-1} . R_{a}$ is the aerodynamic resistance reflecting the turbulent transport to the ocean surface, which is a function of sea-surface roughness, wind speed and atmospheric stability. $R_{b}$ is the quasilaminar boundary layer resistance that describes the quasilaminar transport through a thin layer of air in contact with the surface and which is, in addition to the above listed parameters that control $R_{a}$, also a function of the diffusivity of the trace gas of interest. Finally, $R_{c}$ is the surface resistance that reflects the surface uptake efficiency which can be controlled by physical, chemical and biological processes. Over water, this concept has been expanded to include turbulent and molecular sublayers in both fluids (Liss, 1973). It is also common to use transfer velocities (i.e., reciprocal of resistances) to characterize the sublayer transfers (Fairall et al., 2000).

Published by Copernicus GmbH on behalf of the European Geosciences Union. 
Observed deposition velocities are reported in the literature with values ranging from $V_{d} \sim 0.01$ to $0.12 \mathrm{~cm} \mathrm{~s}^{-1}$ for ocean water and $0.01-0.1 \mathrm{~cm} \mathrm{~s}^{-1}$ for fresh water (Ganzeveld et al., 2007 $)$. This literature gives little details on the chemical, biological and physical water properties during the observations. Currently, values on the order of $V_{d}=0.013$ to $0.05 \mathrm{~cm} \mathrm{~s}^{-1}$ are used in atmospheric chemistry models (Ganzeveld and Lelieveld, 1995; Shon and Kim, 2002). Because the observations do not yield a consensus on wind speed dependency, the same ozone surface resistance is typically applied to all of the world's oceans and wind conditions.

In general, the deposition of ozone involves both turbulent and molecular diffusive plus chemical processes in air and water. If atmospheric chemical reactions are negligible (see Lenschow, 1982; Geernaert et al., 1998; Sorensen et al., 2005, for counter examples), then the atmospheric part of the problem can be treated with standard similarity theory (Fairall et al., 2000). In the near-surface region, vertical turbulent diffusion in both fluids exhibits near-linear height/depth dependence associated with restriction of eddies by the presence of the boundary. Furthermore, the viscosity of a turbulent fluid causes dissipation of the turbulence that is more intense the smaller the turbulent eddy. This leads to a turbulent microscale $\delta_{u} \approx 10 v / u_{*}$ ( $v$ is the fluid kinematic viscosity and $u_{*}$ the friction velocity) such that the spectrum of turbulent fluctuations for eddies smaller than $\delta_{u}$ is exponentially attenuated. Because of this suppression of turbulent eddies near the boundary, ozone entering the water from the air is initially transported away from the interface solely by molecular diffusion. This interfacial region dominated by molecular transport is called the molecular sublayer. The time scale associated with random molecular transport over a distance $\delta$ is $t_{D}=\delta^{2} / D_{x}$ where $D_{x}$ is the molecular diffusivity of the gas, $X$, in the fluid. If the time scale of some chemical reaction for $X$ within the fluid can be characterized by $1 / a$, then in the absence of turbulent effects, we expect the reaction to be substantially completed within a distance $\delta=\left[D_{x} / a\right]^{1 / 2}$. Because $\delta_{u}$ is about $10^{-3} \mathrm{~m}$, this simple scale analysis suggests that for ozone turbulent transport effects need not be considered when $a$ exceeds about $100 \mathrm{~s}^{-1}$.

Garland et al. (1980) used a horizontally homogeneous conservation equation to link the oceanic chemical reactivity of ozone to the oceanic deposition resistance by solving the case where $\delta \ll \delta_{u}$ (i.e., turbulent diffusion was neglected). Schwartz (1992) discussed the more general problem of the balance of solubility and aqueous reaction kinetics from the point of view of chemical enhancement of solubility for reversible reactions for a variety of gases. Chemical enhancement refers to an apparent increase of the solubility of the

\footnotetext{
${ }^{1}$ Ganzeveld, L., Helmig, D., Fairall, C. W., and Pozzer, A.: Biogeochemistry and water-side turbulence dependence of global atmospheric-ocean ozone exchange, Global Biogeochem. Cycles, in preparation, 2007.
}

gas by reactions in the water. The context for that discussion was the stagnant film model, which is equivalent to neglecting turbulent transport in the aqueous phase. In the irreversible limit, Schwartz's results for ozone reduce to the Garland result. More recently, Chang et al. (2004) expanded the scope to combine molecular diffusive - chemical and turbulent diffusive - chemical processes as parallel resistances. In this approach, the oceanside stagnant film resistance of Garland et al. (1980), $R_{g}$, acts independently and in parallel with a Schmidt-number dependent oceanic resistance, $R_{w}$, taken from Wanninkhof (1992) but which includes a chemical enhancement factor: $R_{c}=\left(1 / R_{w}+1 / R_{g}\right)^{-1}$. Chang et al. (2004) also discuss various oceanic chemicals that are expected to be the reacting agent (iodide being the strongest candidate).

Recent research on ocean-atmosphere gas and energy exchange has resulted in improved models that describe the dependencies of deposition on atmospheric and oceanic processes from a more fundamental perspective (Fairall et al., 2000; Hare et al., 2004). In this paper, we will apply this formalism to a trace atmospheric gas that reacts chemically in the ocean. We extend the approach of Garland et al. (1980) to the case where not all of the gas reacts within the molecular sublayer. Whereas Chang et al. (2004) postulate that the deposition velocity is a combination of independent parallel resistances, we derive the deposition velocity analytically from the fundamental conservation equations (albeit in simplified form). Their approach includes a characteristic reaction constant, $a$, plus the chemical enhancement factor, $\beta$; in our approach, the "enhancement" effect is a natural consequence of the solutions to the budget equation.

\section{Conservation equation}

Using the notation from the 2000 Fairall et al. paper, the budget equation for the mass concentration of some chemical, $X_{w}$, in water is

$\partial X_{w} / \partial t+\boldsymbol{U} \cdot \nabla X_{w}=-\frac{\partial\left[\overline{w^{\prime} x_{w}^{\prime}}-D_{x w} \partial X_{w} / \partial z\right]}{\partial z}-a X_{w}$

where $z$ is the vertical coordinate (distance from the interface, i.e., depth for the ocean), $U$ the mean horizontal flow, $\overline{w^{\prime} x_{w}^{\prime}}$ the turbulent flux (positive downward), $D_{x w}$ the molecular diffusivity of $X$ in water, and the last term is the loss rate of $X_{w}$ due to reactions with some chemical $Y_{w}$. We represent the turbulent flux in terms of an eddy diffusion coefficient, $\overline{w^{\prime} x_{w}^{\prime}}=-K \frac{\partial X_{w}}{\partial z}$, where $K(z)$ is the turbulent eddy diffusivity,

$\partial X_{w} / \partial t+\boldsymbol{U} \cdot \nabla X_{w}=-\frac{\partial\left[-\left(D_{x w}+K(z)\right) \partial X_{w} / \partial z\right]}{\partial z}-a X_{w}$

The reactivity $a=C_{x y} Y_{w}$, where $Y_{w}$ is the concentration of the reacting chemical and $C_{x y}$ the reaction rate constant. Assuming $Y_{w}$ is constant with depth, we can move the reaction $\left(a * X_{w}\right)$ term inside the $z$-derivative and associate this with 
a flux variable, $F_{x w}$, which, in dynamic equilibrium, is constant:

$$
-\left[D_{x w}+K(z)\right] \partial X_{w} / \partial z+a \int_{0}^{z} X_{w}(z) d z=F_{x w} .
$$

This flux variable is the sum of transport (mixing) fluxes by molecular diffusion, $F_{x D}$, and turbulent diffusion, $F_{x T}$, plus an apparent flux associated with the decreasing concentration of ozone as it enters and penetrates the ocean and is destroyed by reaction with $Y$.

To apply Eqs. (2) and (3) to the case of an inert or weakly reacting gas, we let $a=0$. This simplifies the analysis because we can directly write an equation for the concentration difference:

$\frac{\partial X_{w}}{\partial z}=\frac{F_{x w}}{D_{x w}+K(z)}$

$X_{w s}-X_{w}\left(z_{r}\right)=F_{x w} \int_{0}^{z_{r}} \frac{d z}{D_{x w}+K(z)}$

$=F_{x w}\left\{\int_{0}^{\delta_{u}} \frac{d z}{D_{x w}+K(z)}+\int_{\delta_{u}}^{z_{r}} \frac{d z}{D_{x w}+K(z)}\right\}$

From Eq. (4b) the resistance law analogy becomes apparent where the total resistance $R_{x w}$ (which is the inverse of the transfer velocity, $V_{x w}$ ) is the sum of the molecular diffusion sublayer resistance, $R_{x w m}$, and the turbulent layer, $R_{x w t}$,

$X_{w s}-X_{w}=F_{x w} R_{x w}=F_{x w}\left(R_{x w m}+R_{x w t}\right)=F_{x w} / V_{x w}$

Here $R_{x w m}$ is the integral over the velocity diffusion sublayer and $R_{x w t}$ the integral from the top of the turbulent layer to the reference depth.

We can write a similar equation for the transport of $X$ in the atmosphere (Fairall et al., 2000). Conventionally, the atmospheric equation is defined with the vertical ordinate as height above the interface and transport fluxes are defined positive upward so that the flux in the atmosphere associated with deposition to the surface is given by

$F_{x a}=-V_{d x} X_{a}=-F_{x w s}$

where $X_{a}$ is the mass concentration at some reference height in the atmosphere and $F_{x w s}$ is the flux into the water at the air-water interface. In equilibrium, the oceanic total flux (remember, this flux is the sum of local transport and accumulated loss of $X$ via chemical reaction) is independent of depth, so $F_{x w s}=F_{x w}$. As in Eq. (5) the atmospheric flux can be characterized by an atmospheric-side transfer velocity and the difference in the concentration at the interface and the reference height

$F_{x a}=V_{x a}\left(X_{a s}-X_{a}\right)=\frac{\left(X_{a s}-X_{a}\right)}{\left(R_{x a m}+R_{x a t}\right)}$
In the absence of atmospheric chemical reactions, the $R_{a}=R_{x a m}$ and $R_{b}=R_{x a t}$ terms would follow from integrating Eq. (4b) with the normal similarity relations (Fairall et al., 2000). A similar relationship applies for the ocean side

$F_{x a}=-F_{x w s}=-V_{x w}\left(X_{w s}-X_{w}\right)$

Using the solubility relationship $X_{w s}=X_{a s} * \alpha_{x}$, where $\alpha_{x}$ is the dimensionless solubility of $X$, we can eliminate the surface concentrations and derive a general flux relationship in terms of the atmospheric and oceanic gas concentrations

$F_{x a}=\frac{\left(X_{w} / \alpha_{x}-X_{a}\right)}{\left(R_{a}+R_{b}\right)+\left(\alpha_{x} V_{x w}\right)^{-1}}$

Note that Eq. (9) can be applied even if there is a chemical reaction in the ocean, but the interpretation of the atmospheric resistance as a sum of molecular and turbulent diffusion sublayer components only follows directly from the budget equation for a non-reactive atmosphere. For the deposition problem where ozone is destroyed by chemical reaction in the ocean, $X_{w}=0$, it follows that

$R_{c}^{-1}=\alpha_{x} V_{x w}=\alpha_{x} F_{x w s} / X_{w s}$

$V_{d x}=\left(R_{a}+R_{b}+R_{c}\right)^{-1}$

\section{Oceanic transfer velocity from the budget equation}

In this section we will solve the basic conservation equation for ozone entering the ocean from the atmosphere. To simplify the notation, we will drop the $w$ subscripts in this section because it deals only with oceanic processes.

\subsection{Negligible turbulence solution}

In the limit that the reaction is so strong that the profile of $X_{w}$ becomes negligible within the oceanic molecular sublayer (besides ozone, other obvious examples include $\mathrm{HNO}_{3}$ and $\mathrm{SO}_{2}$; the paradox that ozone is both strongly reacting in the ocean and is ocean-transfer limited is caused by its weak solubility), we can neglect the $K$ term and write

$D_{x} \frac{\partial^{2} X}{\partial z^{2}}-a X=0$

Assuming that the concentration of $Y$ is much larger than $X$ so that it remains effectively constant, the solution is (Garland et al., 1980)

$X=X_{s} \exp \left[-\sqrt{\frac{a}{D_{x}}} z\right]$

where $X_{S}$ is the concentration of $X$ at the water surface. The diffusive flux at any depth in the fluid is

$$
\begin{aligned}
F_{x D}(z) & =-D_{x} \frac{\partial X}{\partial z}=-D_{x} \frac{\partial}{\partial z}\left\{X_{s} \exp \left[-\sqrt{\frac{a}{D_{x}}} z\right]\right\} \\
& =X_{s} \sqrt{a D_{x}} \exp \left[-\sqrt{\frac{a}{D_{x}}} z\right]
\end{aligned}
$$




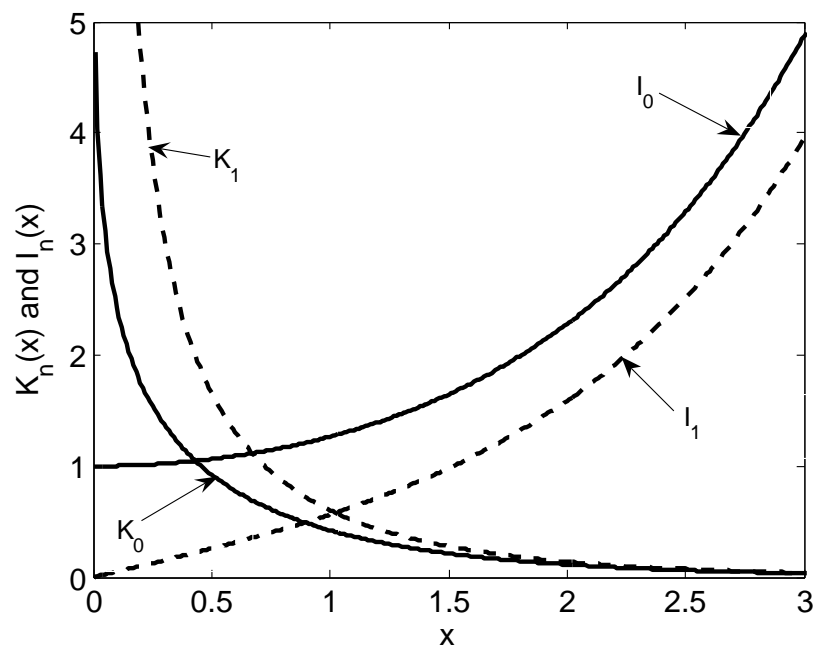

Fig. 1. Graphical representations of the Modified Bessel Functions of order 0 and 1 for the dimensionless variable, $x$ (order 0 : solid line and order 1: dashed line).

The diffusive flux is a function of depth but at the interface $(z=0)$

$F_{x D}(0)=F_{x s}=X_{s} \sqrt{a D_{x}}$

From Eq. (10) it immediately follows that

$V_{x w}=F_{x s} / X_{s}=\sqrt{a D_{x}}$

\subsection{Non-negligible turbulence solution}

To consider the turbulent transport case, we first specify a simple form for the turbulent eddy diffusivity that is obtained from surface-layer similarity scaling (Fairall et al., 2000) $K(z)=\kappa u_{*} z$. Here we have neglected buoyancy (stability effects), $\kappa=0.4$ is the von Karman constant, and $u_{*}$ is the friction velocity in the ocean surface layer. If we do not neglect turbulent transport, then Eq. (2) becomes

$\frac{\partial}{\partial z}\left[\left(D_{x} / \kappa u_{*}+z\right) \frac{\partial X}{\partial z}\right]-\frac{a}{\kappa u_{*}} X=0$

If we transform to $\mathrm{y}^{2}=\left(D_{x} / \kappa u_{*}+z\right)$, then the solutions are modified Bessel functions of zero order (Geernaert et al., 1998)

$X=A I_{0}(\xi)+B K_{0}(\xi)$

$\xi^{2}=\frac{4 a}{\kappa u_{*}}\left(z+\frac{D_{x}}{\kappa u_{*}}\right)$

Details on modified Bessel functions of order $n, I_{n}$ and $K_{n}$, can be found in Abramowitz and Stegun (1964); examples for $n=0$ and 1 are shown in Fig. 1 . To determine $A$ and $B$, we invoke the boundary conditions. If $a$ is uniformly distributed throughout the ocean, the boundary conditions are defined at the interface $(z=0)$ and infinitely deep in the ocean $(z \rightarrow \infty)$

Deep Ocean: $\quad X(z) \rightarrow 0 ; z \rightarrow \infty$
Surface: $\quad-\left[D_{x}+K(z)\right] \frac{\partial X}{\partial z}=F_{x s} ; z \rightarrow 0$

Because $I_{0}$ becomes large as $z$ increases, condition Eq. (18a) implies $A=0$. If we assume that $X=B^{*} K_{0}(\xi)$. In terms of $K_{0}$, the total mixing component of the flux is

$$
\begin{aligned}
F_{x M} & =F_{x D}+F_{x T}=-\left(D_{x}+\kappa u_{*} z\right) \frac{\partial X}{\partial z} \\
& =-B\left(D_{x}+\kappa u_{*} z\right) \frac{\partial K_{0}(\xi)}{\partial z}
\end{aligned}
$$

Writing this in terms of the variable $\xi$, we use the property of $K_{0}$ so that $-\xi \frac{\partial K_{0}}{\partial \xi}=K_{1}$ to describe the mixing component as a function of depth

$$
\begin{aligned}
\frac{F_{x M}}{B} & =-\frac{\left(\kappa u_{*}\right)^{2}}{4 a} \xi^{2} \frac{\partial K_{0}(\xi)}{\partial \xi} \frac{\partial \xi}{\partial z} \\
& =-\frac{\left(\kappa u_{*}\right)^{2}}{4 a} \xi^{2} \frac{\partial K_{0}(\xi)}{\partial \xi} \frac{2 a}{\kappa u_{*}} \xi^{-1}=\frac{\kappa u_{*}}{2} \xi K_{1}(\xi)
\end{aligned}
$$

We then determine the constant $B$ by evaluating Eq. (20) at the surface (condition 18b)

$B=\frac{2 F_{x s} / \kappa u_{*}}{\xi_{0} K_{1}\left(\xi_{0}\right)}$

where

$\xi_{0}=\frac{2}{\kappa u_{*}} \sqrt{a D_{x}}$

Determination of $B$ allows us to explicitly write the equation for the profile of $X$ in the water. We substitute Eq. (21) in Eq. (17a) with $A=0$ :

$X(z)=\frac{2 F_{x s} / \kappa u_{*}}{\xi_{0} K_{1}\left(\xi_{0}\right)} K_{0}(\xi)$

And the profile of the mixing component of the flux

$F_{x M}(z)=F_{x s} \frac{\xi K_{1}(\xi)}{\xi_{0} K_{1}\left(\xi_{0}\right)}$

Notice that Eq. (24) describes how $F_{x M}(z)$ declines as the gas is absorbed; the decline of the mixing flux is balanced by destruction of $X$ by chemical reaction. A bit of algebra shows that Eq. (3) can be written

Total Flux $=\left[\xi K_{1}(\xi)+\int_{\xi_{0}}^{\xi} \xi K_{0}(\xi) d \xi\right] \frac{F_{x s}}{\xi_{0} K_{1}\left(\xi_{0}\right)}$

The first term is the transport (turbulent plus molecular diffusion) and the second is the loss by chemical reaction. Far into the water, the transfer term becomes 0 and the flux entering the fluid has all been consumed:

$F_{x s}=a \int_{0}^{\infty} X(z) d z=\frac{\kappa u_{*}}{2} B \int_{\xi_{0}}^{\infty} K_{0}(\xi) \xi d \xi$ 
Through the properties of Bessel functions, $\xi K_{0}(\xi)=-\frac{\partial\left(\xi K_{1}(\xi)\right)}{\partial \xi}$, Eq. (26) provides an alternate method to relate $B$ to the surface flux.

The water-side transfer velocity is obtained simply from using Eq. (23) in Eq. (15)

$V_{x w}=\frac{\kappa u_{*}}{2} \frac{\xi_{0} K_{1}\left(\xi_{0}\right)}{K_{0}\left(\xi_{0}\right)}=\sqrt{a D_{x}} \frac{K_{1}\left(\xi_{0}\right)}{K_{0}\left(\xi_{0}\right)}$

The limiting values of Bessel functions are well known, so we can examine Eq. (27) in the limit where $a$ is large; in this case, $\xi_{0}$ is large and the ratio $K_{1} / K_{0}=1$. Thus, we recover the Garland et al. (1980) solution given in Eq. (15). The profile of $X(z)$ in the diffusion sublayer is given by Eq. (4) and the concentration of $X$ approaches 0 for $z>D_{x} / \kappa u_{*}$.

For small values of $a$, we find that

$V_{x w} \rightarrow-\frac{\kappa u_{*}}{2} \ln \left(\frac{2}{\kappa u_{*}} \sqrt{a D_{x}}\right)$

In this regime the profile of $X$ is linear in the diffusion sublayer and then logarithmic in $z$ and approaches 0 for $z \approx \kappa u_{*} / 4 a=\delta_{T}$. The transition between strongly and weakly reacting regimes occurs for $\xi_{0} \approx 1$

$a_{\text {crit }}=\frac{\left(\kappa u_{*}\right)^{2}}{4 D_{x}}$

Typical open ocean values $\left(\kappa u_{* w} \approx 0.0037 \mathrm{~ms}^{-1}\right.$ and $D_{\text {ozone }} \approx 3.010^{-9} \mathrm{~m}^{2} \mathrm{~s}^{-1}$ ) in Eq. (29) give the transition around $a_{\text {crit }} \approx 1000 \mathrm{~s}^{-1}$. Ganzeveld et al. $(2007)^{1}$ find the value of $a$ for ozone considering the Iodide-DMS-alkene chemistry never exceeds $1000 \mathrm{~s}^{-1}$. Introducing highlyparameterized DOM-O3 chemistry based on the chlorofyll concentrations, it is exceeded for some confined regions close to coasts. If $a$ significantly exceeds $a_{\text {crit }}$, then ozone is consumed within the oceanic diffusion sublayer. The dimensionless parameter $\xi_{0}$ defined in Eq. (22) is, in fact, the ratio of the chemo-molecular diffusive scale $\delta_{D}$ defined in the Introduction and the chemo-turbulent diffusive scale $\delta_{T}$ defined above.

\subsection{Two-layer reactivity (surfactant) solution}

In this section we examine a more complicated vertical distribution of reactivity designed to mimic assumed properties of a highly reactive surfactant. A surfactant may be a hydrophilic material that tends to have much enriched concentration at the surface or a soluble compound that influences some surface property of seawater (e.g., viscosity or surface tension). We do not say what this surfactant is but specify its properties as having reactivity $a$ beginning at the interface and down to a depth $\delta$ relative to some background reactivity $a_{o}$ that is present everywhere. Here we consider a two-layer solution

Layer I: $\quad 0<z<\delta$

where reactivity $=a+a_{o} \quad X(z)=A_{I} I_{0}(\xi)+B_{I} K_{0}(\xi)$
Layer II: $\quad z>\delta$

where reactivity $=a_{o} X(z)=B_{I I} K_{0}(\xi)$

In layer I the solutions are described by Eq. (17a) but $A$ is not 0 ; in layer II $A=0$.

In order to find the values of the three coefficients, we must match three boundary conditions: (1) the flux at the surface, (2) the continuity of concentration at the I-II boundary, and (3) the surface flux must equal the total absorption of $X$ by reaction in the medium. For the general form of $X(z)$, the transport flux is

$F_{x M}(z)=\frac{\kappa u_{*}}{2}\left[-A \xi I_{1}(\xi)+B \xi K_{1}(\xi)\right]$

The three boundary conditions can be written as follows:

$$
\begin{aligned}
& -A_{I} \xi_{0} I_{1}\left(\xi_{0}\right)+B_{I} \xi_{0} K_{1}\left(\xi_{0}\right)=\frac{2 F_{x s}}{\kappa u_{*}} \\
& A_{I} I_{0}\left(\xi_{\delta}\right)+B_{I} K_{0}\left(\xi_{\delta}\right)-B_{I I} K_{0}\left(\xi_{\delta}\right)=0 \\
& A_{I}\left(a+a_{0}\right) \int_{0}^{\delta} I_{0}(\xi) d z+B_{I}\left(a+a_{0}\right) \int_{0}^{\delta} I_{0}(\xi) d z \\
& +B_{I I} a_{0} \int_{\delta}^{\infty} K_{0}(\xi) d \xi=F_{x s}
\end{aligned}
$$

Alternatively, a flux continuity condition at the I-II interface can be substituted for any one of these equations. The three relationships from Eq. (32) can be written as the product of a $3 \mathrm{X} 3$ matrix times a coefficient vector $=$ flux vector $(\mathbf{H} * \boldsymbol{A}=\boldsymbol{F})$ :

$$
\left(\begin{array}{lll}
h_{11} & h_{12} & h_{13} \\
h_{21} & h_{22} & h_{23} \\
h_{31} & h_{32} & h_{33}
\end{array}\right) *\left(\begin{array}{l}
A_{I} \\
B_{I} \\
B_{I I}
\end{array}\right)=\left(\begin{array}{l}
\frac{2 F_{x s}}{\kappa u_{*}} \\
0 \\
F_{x s}
\end{array}\right)
$$

where the $h_{i j}$ coefficients come from the terms in Eq. (32). The coefficients are found by inverting the $\mathbf{H}$ matrix, $\boldsymbol{A}=\mathbf{H}^{-1} * \boldsymbol{F}$. Once the coefficients are obtained, the waterside transfer velocity is given by

$$
V_{x w}=\sqrt{\left(a+a_{0}\right)} D_{x} \frac{\left[-A_{I} I_{1}\left(\xi_{0}\right)+B_{I} K_{1}\left(\xi_{0}\right)\right]}{\left[A_{I} I_{0}\left(\xi_{0}\right)+B_{I} K_{0}\left(\xi_{0}\right)\right]}
$$

\section{Discussion}

The ocean-side transfer velocity given in Eq. (27) depends principally on the forcing (atmospheric friction velocity), the molecular diffusivity (or Schmidt number), and the reactivity. The effective "pull" on the atmosphere also requires the solubility. A simple example of sensitivity to forcing and reactivity is shown in Fig. 2. Here we plot a family of curves (each curve for a different value of atmospheric friction velocity $u_{* a}$ ) for the dependence of $\alpha_{x} V_{x w}$ as a function of reactivity. The atmospheric friction velocity is roughly proportional to wind speed with a value of $0.35 \mathrm{~ms}^{-1}$ at a $10-\mathrm{m}$ 


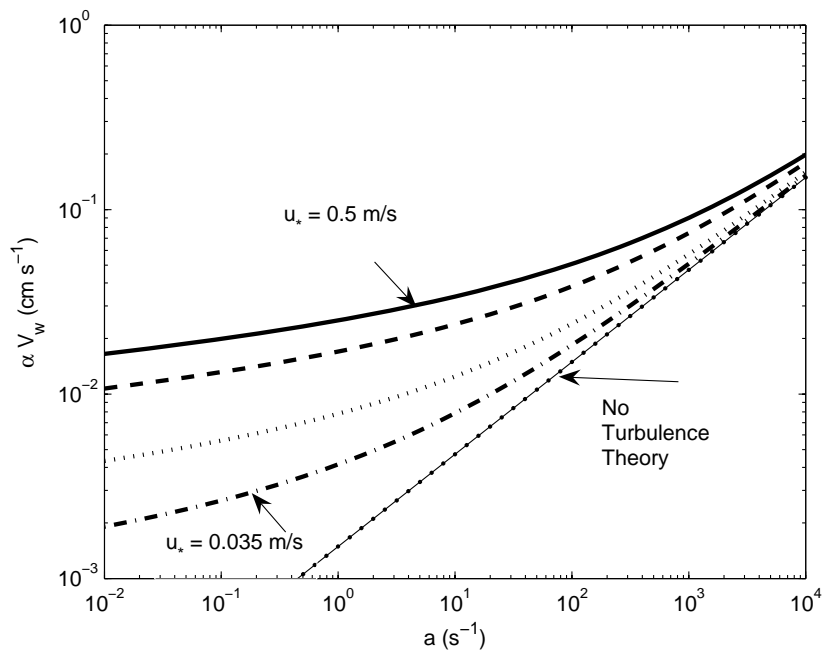

Fig. 2. Water-side transfer velocity (multiplied by solubility) for ozone from Eq. (27) as a function of reactivity, $a$. The individual curves are for different values of friction velocity: solid: $u_{* a}=0.5 \mathrm{~ms}^{-1}$; dashed: $u_{* a}=0.3 \mathrm{~ms}^{-1}$; dotted: $u_{* a}=0.1 \mathrm{~ms}^{-1}$; dashdot: $u_{* a}=0.035 \mathrm{~ms}^{-1}$. The dots with the thin line are the noturbulence solution.

wind speed of $10 \mathrm{~ms}^{-1}$. If we assume the atmospheric stress drives an equal turbulent stress in the ocean, then the oceanic friction velocity follows from the ratio of the densities

$u_{* w}=\sqrt{\frac{\rho_{a}}{\rho_{w}}} u_{* a} \approx u_{* a} / 30 \approx 0.0012 U_{10}$

The curves in Fig. 2 are bounded on the bottom by the noturbulence (stagnant film) theory of Garland et al. (1980). The family of curves spans wind speeds from about 1.0 to $15 \mathrm{~ms}^{-1}$. For strong winds the oceanic transfer velocity is much more weakly dependent on $a$. Regarding the total atmospheric deposition velocity, interpretation of the implications of Fig. 2 requires specification of the atmospheric transfer. We use the NOAA-COARE gas transfer model (Fairall et al., 2000; Hare et al., 2004)

$R_{x a}=R_{a}+R_{b}=\left[C_{d}^{-1 / 2}+13.3 S_{c a}^{1 / 2}-5+\frac{\log \left(S_{c a}\right)}{2 \kappa}\right] / u_{* a}$

where $C_{d}$ is the momentum drag coefficient at the reference height and $S_{c a}$ the Schmidt number for ozone in air (about 1). In Eq. (36) the $C_{d}$ term represents $R_{a}$ and the remaining terms represent $R_{b}$. For an atmospheric reference height of $10 \mathrm{~m} \mathrm{C}_{d}^{-1 / 2} \approx 28$; at a wind speed of $10 \mathrm{~ms}^{-1}$ the atmospheric resistance $R_{a}+R_{b} \approx 100 \mathrm{sm}^{-1}$, implying a transfer velocity of about $1.0 \mathrm{cms}^{-1}$. Typical observed ozone total deposition values are on the order of $0.05 \mathrm{cms}^{-1}$ (total $R=2000 \mathrm{sm}^{-1}$ ), so we know that $R_{c}$ dominates the total transfer resistance. From Fig. 2 we can see that $0.05 \mathrm{cms}^{-1}$ corresponds to $a \approx 10^{3} \mathrm{~s}^{-1}$.

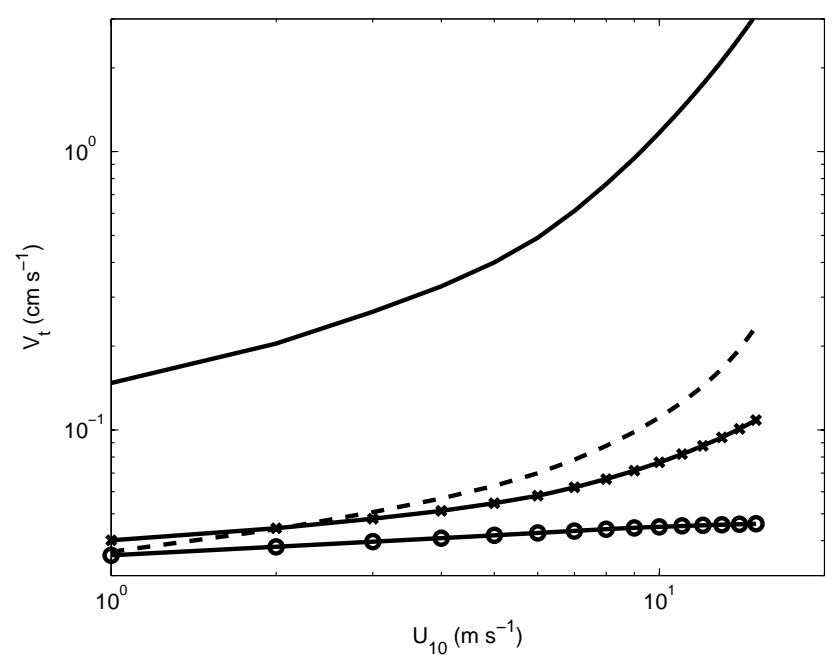

Fig. 3. Total deposition velocity as a function of wind speed for ozone using Eq. (10) with $a=1000 \mathrm{~s}^{-1}$. The solid line is the atmospheric component, $R_{x a}$, from Eq. (36). The dashed line is $V_{d}$ combining Eq. (36) with Eq. (27) for $V_{x w}$; the line with circle symbols is $V_{d}$ combining Eq. (36) with stagnant film result (Eq. 15); the line with x's is $V_{d}$ from Chang et al. (2004).

Because $V_{d}$ for ozone is usually dominated by the oceanic component, it is clear from Fig. 2 that ocean turbulence probably plays a significant role in the variability of ozone deposition. This conclusion follows from the observed wind-speed dependence of $V_{d}$ because the stagnant film result (Eq. 16) is independent of wind speed. An alternative explanation is that $a$ systematically increases with wind speed, which contradicts the conventional wisdom that surfactants are more prevalent in light winds. Figure 3 shows wind speed dependencies obtained using Eq. (27) in Eq. (9) when specifying $a=10^{3} \mathrm{~s}^{-1}$. Note the atmospheric transfer velocity (solid line) is about 10 times larger than the effective oceanic velocity. Thus, for this value of $a$, the ocean is the dominant bottleneck to transfer; $a$ would have to be two orders of magnitude larger for the oceanic and atmospheric resistances to be comparable. The wind-speed dependence of the no-turbulence theory for $V_{d}$ is very weak because it enters only through the atmospheric component (Eq. 15), which does not depend on $u_{*}$. The model of Chang et al. (2004), which empirically incorporates ocean turbulence in a less rigorous way, gives results that are fairly similar to Eq. (27).

The surfactant case has been examined by specifying a background value $a_{0}=10^{-4} \mathrm{~s}^{-1}$ to the result of a thin layer of thickness $10^{-5} \mathrm{~m}$ of surfactant as suggested by Schwartz (1992) (i.e. deep layers are not required). Figure 4 shows the dependence of $\alpha_{x} V_{x w}$ as a function of surfactant reactivity for $u_{* a}=0.035 \mathrm{~ms}^{-1}$. The one-layer (27) solution that assumes the reactant is deeply distributed and the twolayer (34) solution become comparable for $a$ on the order of $100 \mathrm{~s}^{-1}$; for $u_{* a}=0.5 \mathrm{~ms}^{-1}$ (not shown) they become com- 


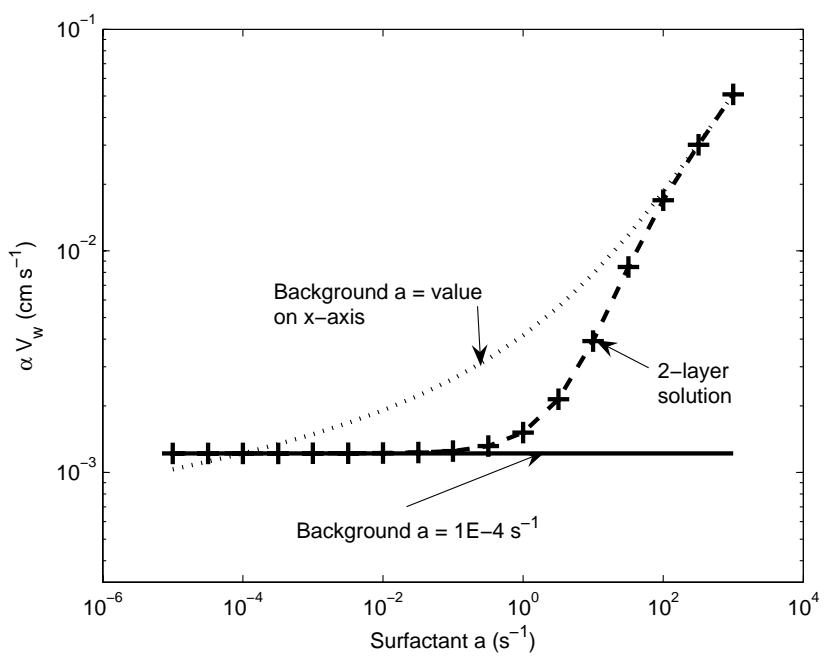

Fig. 4. Water-side transfer velocity (multiplied by solubility) for ozone as a function of reactivity, $a$, for $u_{* a}=0.035 \mathrm{~ms}^{-1}$. The flat solid line denotes the velocity with a fixed background at $a=a_{0}=10^{-4}$; the dotted line denotes the velocity computed with Eq. (27) with $a$ taking the values on the x-axis. The dashed line with plus symbols denotes the velocity computed using Eq. (34) with a surfactant layer $10^{-5} \mathrm{~m}$ thick with reactivity on the $\mathrm{x}$-axis which is added to the background value.

parable for $a$ on the order of $1000 \mathrm{~s}^{-1}$. This suggests that observed values of ozone deposition velocities could be the result of a thin layer of surfactant (i.e., deep layers are not required) as suggested by Schwartz (1992).

The one-layer ozone deposition velocity parameterization has been coded in Matlab and Fortran90 in a form that is easily paired with the NOAA-COARE bulk flux algorithm (Fairall et al., 2003). In addition to the normal near-surface variables needed for bulk fluxes (i.e., in the COARE algorithm), inputs are required for $\alpha_{x}, a$, and $S_{c w}$. For illustration we have computed transfer velocities from a recent field program on the NOAA Ship Ronald H. Brown that was conducted off the coast of New Hampshire in July and August 2004. Further details on the measurements and the field program are available at http://www.etl.noaa.gov/programs/ 2004/neaqs/flux/. The bulk meteorological variables measured from the ship are input to the NOAA-COARE flux algorithm and then the meteorological fluxes are used to compute the ozone deposition velocity. Deposition velocities are computed for a 16-day period after specifying $\alpha_{x}=0.3$, $a=10^{3} \mathrm{~s}^{-1}$, and $S_{c w}=500$ (Fig. 5). The no-turbulence model shows little variation except for occasional periods of lighter winds and strong atmospheric stability (warm air over cool water) where hydrostatic stability effects suppress both $u_{*}$ and the atmospheric transfer.

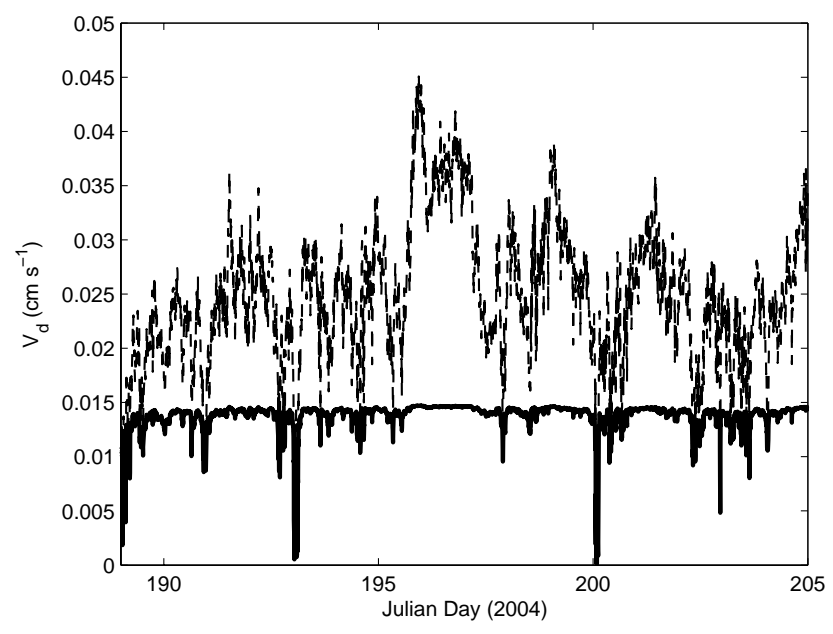

Fig. 5. Time series of ozone deposition velocity computed from bulk meteorological measurements from a recent cruise of the NOAA Ship Ronald H. Brown off New England in July and August 2004. The thick line is $V_{d}$ computed with using Eq. (15) with Eq. (36), which neglects turbulent transport in the ocean; the thin dashed line is Eq. (27) with Eq. (36), which includes turbulent transport in the ocean. Ozone variables are specified as $\alpha_{x}=0.3$, $a=10^{3} \mathrm{~s}^{-1}$, and $S_{c w}=500$.

\section{Conclusion}

Starting from the fundamental conservation equation, we have derived relationships for the deposition velocity of ozone to the ocean that accounts for the oceanic chemical destruction. This work has several implications for interpretation and planning of field observations. Typical deposition values quoted in the literature imply that the atmospheric resistance is small compared to the oceanic resistance. Furthermore, the atmospheric resistance is well-characterized after decades of study of temperature, moisture, and trace gas investigations. Thus, oceanic mechanisms dominate the uncertainty in the parameterization of ozone deposition to the sea. This uncertainty involves not only the normal complexity of oceanic mechanisms such as breaking waves and oceanic bubbles (see Fairall et al., 2000) but the additional uncertainty associated with variability in the near-surface chemical reactions. The value of reactivity $\left(a=10^{3} \mathrm{~s}^{-1}\right)$ that is consistent with observations of ozone deposition velocity suggest a thin ozone penetration depth in the ocean that could be provided by a surfactant microlayer. However, our results show that even in that case oceanic turbulent mixing will still play a role in deposition (e.g., Fig. 3).

It is clear that significant progress on ozone deposition in the future will require field observations that combine direct covariance ozone measurements with chemical and physical measurements in the ocean in a variety of locations that span reasonable ranges of variables. Furthermore, accurate global modeling/assessment of ozone fluxes will probably require 
global characterizations of near-surface chemistry relevant to ozone oceanic transfer (see Ganzeveld et al., 2007 ${ }^{1}$ ).

The algorithms and data used in this example are available at the following ftp site:

ftp://ftp.etl.noaa.gov/user/cfairall/bulkalg/gasflux/ozone/.

\section{List of Symbols}

$a \quad$ Chemical reactivity in the ocean $\left(\mathrm{s}^{-1}\right)$

$a_{\text {crit }} \quad$ Value of a where molecular and turbulent diffusive mechanisms are comparable

$t \quad$ Time (s)

$u_{*} \quad$ Friction velocity; $u_{*}=\sqrt{-\overline{w^{\prime} u^{\prime}}}\left(\mathrm{ms}^{-1}\right)$

$u_{* a} \quad$ Friction velocity for air

$u_{* w} \quad$ Friction velocity for water

$u^{\prime} \quad$ Horizontal velocity turbulent fluctuation

$w^{\prime} \quad$ Vertical velocity turbulent fluctuation

$x^{\prime} \quad$ Turbulent fluctuation of concentration $\mathrm{X}$

$\overline{w^{\prime} x^{\prime}} \quad$ Turbulent covariance (vertical flux) of gas X

$\overline{w^{\prime} u^{\prime}} \quad$ Turbulent stress or covariance of vertical and horizontal velocity fluctuations

$z \quad$ Vertical coordinate, depth in water and height in air (m)

$z_{r} \quad$ Reference depth (or height in air) far from the interface where bulk concentration is measured

A Coefficient the $I_{0}$ Bessel function term

$A_{I} \quad$ Coefficient the $I_{0}$ Bessel function term in layer I (surfactant layer)

$A_{I I} \quad$ Coefficient the $I_{0}$ Bessel function term in layer II (bulk layer)

$B \quad$ Coefficient the $K_{0}$ Bessel function term

$B_{I} \quad$ Coefficient the $K_{0}$ Bessel function term in layer I (surfactant layer)

$B_{I I} \quad$ Coefficient the $K_{0}$ Bessel function term in layer II (bulk layer)

$C_{d} \quad$ Momentum transfer (drag) coefficient

$C_{x y} \quad$ Rate coefficient for reaction of $\mathrm{X}$ and $\mathrm{Y}, \mathrm{a}=\mathrm{C}_{x y} \mathrm{Y}_{w}$

$D_{x} \quad$ Molecular diffusivity for gas $\mathrm{X}\left(\mathrm{m}^{2} \mathrm{~s}^{-1}\right)$

$D_{x a} \quad$ Molecular diffusivity for gas $\mathrm{X}$ in air

$D_{x w} \quad$ Molecular diffusivity for gas $\mathrm{X}$ in water

$F_{x} \quad$ Mass flux variable for gas $\mathrm{X}\left(\mathrm{kgm}^{-2} \mathrm{~s}^{-1}\right)$

$F_{x s} \quad$ Mass flux variable for gas $\mathrm{X}$ at the air-water interface

$F_{x a} \quad$ Mass flux variable for gas $\mathrm{X}$ in air

$F_{x w} \quad$ Mass flux variable for gas $\mathrm{X}$ in water

$F_{x D} \quad$ Mass flux variable for gas $\mathrm{X}$ associated with the molecular diffusion term

$F_{x T} \quad$ Mass flux variable for gas $\mathrm{X}$ associated with the turbulent diffusion term

$F_{x M} \quad$ Mass flux variable for gas $\mathrm{X}$ by mixing, $=F_{x D}+$ $F_{x T}$
$I_{n}, K_{n} \quad$ Modified Bessel functions of order $n$

$K(z) \quad$ Turbulent eddy diffusion coefficient $\left(\mathrm{m}^{2} \mathrm{~s}^{-1}\right)$

$R \quad$ Transfer resistance $\left(\mathrm{sm}^{-1}\right)$

$R_{a} \quad$ Transfer resistance for the atmospheric turbulent sublayer

$R_{b} \quad$ Transfer resistance for the atmospheric molecular sublayer

$R_{c} \quad$ Transfer resistance for the ocean

$R_{g} \quad$ Transfer resistance for the ocean from ozone reactivity from Garland et al. (1980)

$R_{w} \quad$ Transfer resistance for the ocean for mixing from Wanninkhof 1992

$R_{x t} \quad$ Transfer resistance for the atmospheric turbulent sublayer computed via Eq. (4b) $R_{x t a}=R_{a}$

$R_{x m} \quad$ Transfer resistance for the atmospheric molecular sublayer computed via Eq. (4b) $R_{x m a}=R_{b}$

$S_{c x} \quad$ Schmidt number $=v / D_{x}$ for gas $\mathrm{X}$

$S_{c a} \quad$ Schmidt number $=v_{a} / D_{x a}$ for gas $\mathrm{X}$ in air

$S_{c w}$

$U$

Schmidt number $=v_{w} / D_{x w}$ for gas $\mathrm{X}$ in water

Horizontal fluid velocity, wind speed or current speed $\left(\mathrm{ms}^{-1}\right)$

$U_{10} \quad$ Wind speed at a reference height of $10 \mathrm{~m}$

$V_{d} \quad$ Deposition velocity

$V_{d x} \quad$ Deposition velocity for gas $\mathrm{X}$

$V_{x a} \quad$ Transfer velocity for gas $X$ in air, $=1 / R_{x a}$

$V_{x w} \quad$ Transfer velocity for gas $X$ in water, $=1 / R_{x w}$

$X_{a} \quad$ Concentration of $X$ in air $\left(\mathrm{kgm}^{-3}\right)$

$X_{w} \quad$ Concentration of $X$ in water $\left(\mathrm{kgm}^{-3}\right)$

$X_{\text {as }} \quad$ Concentration of $X$ in air at the air-water interface $\left(\mathrm{kgm}^{-3}\right)$

$X_{w s} \quad$ Concentration of $X$ in water at the air-water interface $\left(\mathrm{kgm}^{-3}\right)$

$Y_{w} \quad$ Concentration of the chemical $Y$ that reacts with $X$ in the water $\left(\mathrm{kgm}^{-3}\right)$

$\alpha_{x} \quad$ Dimensionless solubility for gas $X$ in the ocean, $=X_{w s} / X_{a s}$

$\beta \quad$ Chemical enhancement factor where solubility is replaced by $\beta \alpha_{x}$

$\delta \quad$ Transport sublayer thickness (m)

$\delta_{u} \quad$ Turbulent microscale or velocity sublayer thickness

$\delta_{D} \quad$ Chemo-diffusive sublayer thickness for molecular diffusion

$\delta_{T} \quad$ Chemo-diffusive sublayer thickness for turbulent diffusion

$\kappa \quad$ von Karman constant $(=0.4)$

$v \quad$ Kinematic viscosity of a fluid $\left(\mathrm{m}^{2} \mathrm{~s}^{-1}\right)$

$\xi \quad$ Dimensionless depth variable; see Eq. (17) 
Acknowledgements. This work is supported by the NOAA Office of Global Programs (Carbon Cycle program element), the NOAA Health of the Atmosphere program, and NSF award CHE-BE \#0410048. Any opinions, findings, and conclusions expressed in this material are those of the authors and do not necessarily reflect the views of the funding agencies.

Edited by: M. Heimann

\section{References}

Abramowitz, M. and Stegun, I. A.: Handbook of Mathematical functions. Applied Mathematics Series, 55. US Gov. Printing Office, Washington, DC, 1964.

Chang, W., Heikes, B. G., and Lee, M.: Ozone deposition to the sea surface: chemical enhancement and wind speed dependence, Atmos. Environ., 38, 1053-1059, 2004.

Fairall, C. W., Hare, J. E., Edson, J. B., and McGillis, W.: Parameterization and micrometeorological measurements of air-sea gas transfer, Bound.-Layer Meteorol., 96, 63-105, 2000.

Fairall, C. W., Bradley, E. F., Hare, J. E., Grachev, A. A., and Edson, J. B.: Bulk parameterization of air-sea fluxes: Updates and verification for the COARE algorithm, J. Clim., 16, 571-591, 2003.

Ganzeveld, L. and Lelieveld, J.: Dry deposition parameterization in a chemistry general circulation model and its influence on the distribution of reactive trace gases, J. Geophys. Res., 100, 20 999-21 012, 1995.

Garland, J. A., Etzerman, A. W., and Penkett, S. A.: The mechanism for dry deposition of ozone to seawater surfaces, J. Geophys. Res., 85, 7488-7492, 1980.

Geernaert, L. L. S., Geernaert, G. L., Granby, K., and Asman, W. A. H.: Fluxes of soluble gases in the marine atmospheric surface layer, Tellus B, 50, 111-127, 1998.

Hare J. E., Fairall, C. W., McGillis, W. R., Edson, J. B., Ward, B., and Wanninkhof, R.: Evaluation of the National Oceanic and Atmospheric Administration/Coupled-Ocean Atmospheric Response Experiment (NOAA/COARE) air-sea gas transfer parameterization using GasEx data, J. Geophys. Res., 109, C08S11, doi:10.1029/2003JC001831, 2004.
Helmig, D., Oltmans, S. J., Carlson, D., Lamarque, J.-F., Jones, A., Labuschagne, C., Anlauf, K., and Hayden, K.: A review of surface ozone in the polar regions, Atmos. Environ., in press, 2007.

IPCC Climate Change 2001: A report by the Working Group I of the Intergovernmental Panel on Climate Change, http://www. ipcc.ch/, 2001.

Lamarque, J.-F., Hess, P., Emmons, L., Buja, L., Washington, W., and Grainer, C.: Tropospheric ozone evolution between 1890 and 1990, J. Geophys. Res., 110, D08304, doi:10.1029/2004JD005537, 2005.

Lenschow, D. H.: Reactive trace species in the boundary layer from a micrometeorological perspective, J. Meteorol. Soc. Japan, 60 , 472-480, 1982.

Liss, P.: Processes of gas exchange across an air-water interface, Deep-Sea Res., 20, 221-228, 1973.

Oltmans, S. J., Lefohn, A. S., Scheel, H. E., Harris, J. M., Levy II, H., Galbally, I. E., Brunke, E. G., Meyer, C. P., Lathrop, J A., Johnson, B. J., Shadwick, D. S., Cuevas, E., Schmidlin, F. J., Tarasick, D. W., Claude, H., Kerr, J. B., Uchino, O., and Mohnen, V.: Trends of ozone in the tropopsphere, Geophys. Res. Lett., 25, 139-142, 1998.

Schwartz, S.: Factors governing dry deposition of gases to surface water, in: Precipitation Scavenging and Atmosphere-Surface Exchange, vol. 2. edited by: Schwartz, S. and Slinn, S., Hemisphere Publishing Corp., Washington, USA, pp. 789-801, 1992.

Shon, Z.-H. and Kim, N.: A modeling study of halogen chemistry's role in marine boundary layer ozone, Atmos. Environ., 36, 42894298, 2002.

Sorensen, L. L., Pryor, S. C., De Leeuw, G., and Schulz, M.: Flux divergence of nitric acid in the marine atmospheric surface layer, J. Geophys. Res., 110, D15306, doi:10.1029/2004JD005403, 2005.

Vingarzan, R.: A review of surface ozone background levels and trends, Atmos. Environ., 38, 3431-3442, 2004.

Wanninkhof, R.: Relationship between wind speed and gas exchange over the ocean, J. Geophys. Res., 97, 7373-7382, 1992.

Wesely, M. L. and Hicks, B. B.: A review of the current status of knowledge on dry deposition, Atmos. Environ., 34, 2261-2282, 2000 . 